\title{
Relación entre carga laboral y burnout en enfermeras de unidades de diálisis
}

\author{
Silvia Barrios Araya ${ }^{1}-M^{a}$ Cecilia Arechabala Mantuliz² - Victoria Valenzuela Parada ${ }^{3}$
}

${ }^{1}$ Enfermera. Profesor Asistente Escuela de Enfermería Pontificia Universidad Católica. Presidenta de la Sociedad Chilena de Enfermería en Diálisis y Trasplante Renal (SENFERDIALT)

${ }^{2}$ Enfermera. Profesor Asociado. Escuela de Enfermería Pontificia Universidad Católica de Chile

${ }^{3}$ Alumna Egresada. Enfermería Escuela de Enfermería Pontificia Universidad Católica de Chile

\section{Resumen}

La hemodiálisis requiere personal de enfermería con altos niveles de capacitación por su complejidad tecnológica, las peculiaridades del tratamiento y la necesidad de tomar decisiones relevantes para la seguridad del paciente en breve espacio de tiempo: Esto condiciona que estos profesionales estén expuestos a múltiples estresores que están directamente relacionados con la aparición de síndrome de Burnout.

El objetivo de esta revisión fue conocer la relación entre la sobrecarga laboral y los factores que desencadenan la aparición del síndrome Burnout en el personal de enfermería de las unidades de hemodiálisis.

La metodología utilizada fue una búsqueda en bases de datos a través de la colección electrónica del Sistema de Bibliotecas de la Pontificia Universidad Católica de Chile. Cuarenta y tres artículos fueron revisados para analizar los predictores descritos en trabajadores de salud, entre los que destacan: sobrecarga laboral, frecuencia y tiempo dedicado a

Correspondencia:

Silvia Barrios Araya

Escuela de Enfermería Pontificia

Universidad Católica de Chile

E-mail:sbarrios@uc.cl la atención de pacientes, participación en la toma de decisiones, riesgos laborales y riesgo de afectación psíquica y la incorporación constante de nuevas tecnologías y conocimientos.

La evidencia apoya que altos niveles de satisfacción con el puesto de trabajo, relaciones interprofesionales colaborativas, trabajo en equipo, respeto al conocimiento y a la experiencia clínica por parte de los directivos, junto a la posibilidad de participar en la toma de decisiones son factores percibidos por las enfermeras/os como protectores de síndrome de Burnout

PALABRAS CLAVE:

- SÍNDROME DE BURNOUT

- SOBRECARGA LABORAL

- ENFERMERÍA

- HEMODIÁLISIS

\section{Relationship between workload and burnout in nurses in dialysis units}

\section{Abstract}

Haemodialysis requires highly skilled nursing staff because of its technological complexity, the peculiarities of the treatment and the need to make 
relevant decisions for the patient's safety in a short space of time: This means that these professionals are exposed to multiple stress factors that are directly related to the appearance of burnout syndrome.

The purpose of this review was to find out the relationship between excessive workload and the factors that lead to the appearance of burnout syndrome in nursing staff in haemodialysis units.

The methodology used was a database search through the electronic collection of the System of Libraries of the Pontifical Catholic University of Chile. Forty-three articles were reviewed to analyse the predictors described in healthcare workers, notable among which were: excessive workload, frequency and time dedicated to patient care, participation in decision making, occupational risks and risk of psychological impact and the constant incorporation of new technologies and knowledge.

The evidence supports the view that high levels of job satisfaction, collaborative interprofessional relationships, teamwork, management's respect for clinical knowledge and experience, together with the possibility of participating in decision making are factors perceived by nurses as protectors against burnout syndrome.

\section{KEY WORDS:}

- BURNOUT SYNDROME

- EXCESSIVE WORKLOAD

- NURSING

- HAEMODIALYSIS

\section{Introducción}

En los últimos años, el mundo laboral ha experimentado cambios en el contexto socio cultural. Las exigencias y los requisitos del puesto de trabajo en las organizaciones han motivado la aparición de problemas de salud en las personas, entre los que destaca el síndrome de burnout (SB) ${ }^{1}$.
Este trastorno, se conforma como un síndrome tridimensional caracterizado por agotamiento o cansancio emocional que puede manifestarse física y psíquicamente; despersonalización como presencia de sentimientos, actitudes y respuestas negativas distantes y frías hacía otras personas, especialmente hacia los beneficiarios del propio trabajo. Por último, la falta de realización personal o logro que se caracteriza por una dolorosa desilusión para dar sentido a la propia vida y hacia los logros personales con sentimientos de fracaso y baja autoestima $2,3,4,5,47$.

Maslach \& Pines (1977), describen diversos síntomas que caracterizan al burnout entre los que se encuentran: trastornos sicosomáticos, emocionales y conductuales.

Entre los trastornos psicosomáticos se encuentran cefalea; fatiga crónica; desordenes gastrointestinales; mialgias e hipertensión, entre otros. También es posible encontrar manifestaciones emocionales, distanciamiento afectivo con las personas a las que se atiende, deseos de abandonar el trabajo, irritabilidad, y dificultad para concentrarse, produciéndose un descenso del rendimiento lo que se traduce en el cuestionamiento de la propia competencia profesional, que conlleva a un descenso en la autoestima. Además, se encuentran síntomas conductuales, como ausentismo laboral, conducta violenta, abuso de drogas, incapacidad de relajarse y síntomas defensivos que aluden a la negación emocional, cuyas formas más habituales son: negación de las emociones, ironía, atención selectiva y el desplazamiento de sentimientos hacia otras situaciones $0 \operatorname{cosas}^{6,7,7,9,10,11,47}$.

No existen datos claros respecto a la prevalencia de este síndrome. Algunos autores plantean que se trata de una patología altamente prevalente. Un estudio realizado en Chile en personal de salud (médicos, enfermeras y técnicos de enfermería) de un hospital de la Región Metropolitana mostró una prevalencia general de $31 \%$ de SB. Además, la frecuencia de casos sintomáticos aumentó en relación con las horas de trabajo semanal, llegando hasta un $83 \%$ entre los trabajadores con más de 60 horas semanales ${ }^{12,13}$. Una situación similar mostró la investigación de Trucco et al (1999), en un grupo de trabajadores de salud del área privada, encontrando una prevalencia de 45\%. La prevalencia más alta se encontró siendo en el grupo no profesional con un $69,2 \%$ comparado con un $34,3 \%$ en los profesionales ${ }^{14}$. 
Las condiciones laborales y las características del tipo de trabajo desarrollado por las enfermeras en Unidades de Hemodiálisis (UHD) hacen que este sea un grupo de alto riesgo para presentar SB. Esto es debido sin duda, a que trabajan con los aspectos emocionales más intensos del cuidado del paciente, la enfermedad, la muerte, la angustia e incertidumbre ${ }^{15}$.

\section{Marco conceptual}

En Chile, el tratamiento para la Insuficiencia Renal Crónica Terminal (IRCT), más utilizado es la hemodiálisis (HD). Esta, requiere de un personal especializado, tanto profesional como técnico, que según el reglamento establecido por el Ministerio de Salud, la dotación de personal en las UHD es de una enfermera y un técnico de enfermería por cada seis pacientes ${ }^{17}$.

Por otra parte, conviene recordar que los pacientes en $H D$, son cada vez de más edad y con una comorbilidad que condiciona un grado importante de dependencia. Las limitaciones físicas y los cuidados asociados a estos pacientes suponen un aumento significativo de la carga del trabajo del personal de enfermería, que habrá que tener presente para garantizar la calidad de atención a los mismos ${ }^{15,18}$.

La carga de trabajo, se define como las tareas o actividades que habitualmente recaen sobre enfermería; los tiempos de enfermería están directamente relacionados con los cuidados que realiza, por lo que se necesita una alta cualificación en los profesionales que trabajan o quieren hacerlo en el área nefrológica. La investigación muestra que para garantizar estándares mínimos de calidad deben definirse plantillas de trabajo en las diferentes unidades de diálisis, no con parámetros fijos sino adecuando la cantidad total de servicios que se prestan a las necesidades que se detectan ${ }^{19}$.

EI SB implica una pérdida del bienestar en la actividad profesional y del equilibrio emocional, asociado a una vivencia subjetiva más bien depresiva o desesperanzada. Se relaciona con la percepción de que los esfuerzos e inversión de energía no se corresponden con el grado de satisfacción alcanzado. Es importante considerar en este sentido, que la satisfacción laboral se obtiene de las experiencias exitosas, el reconocimiento, el sentimiento de autorrealización y la experiencia común, es decir, la sensación de formar parte de un grupo o institución ${ }^{20,41,42}$.
El objetivo de esta revisión fue conocer la relación entre la sobrecarga laboral y los factores que desencadenan la aparición del SB en el personal de enfermería de las UHD.

\section{Metodología}

La información se categorizó de acuerdo a los predictores de SB descritos en trabajadores de salud, entre ellos se encuentran: sobrecarga laboral, frecuencia y cantidad de tiempo dedicado a la atención de pacientes, participación en la toma de decisiones, riesgo de afectación psíquica y la incorporación constante de nuevas tecnologías y conocimientos ${ }^{16}$. Además de los riesgos del personal de diálisis asociados a la terapia dialítica.

Se realizó una búsqueda en las bases de datos de la colección electrónica del Sistema de Bibliotecas de la Pontificia Universidad Católica de Chile (SIBUC), utilizando las palabras claves: burnout, renal nurses, nursing personal and renal hemodialysis. Los límites fueron artículos del año 2000 en adelante. Se encontraron 104 artículos de estos se seleccionaron 43. Para la selección de las publicaciones los criterios de inclusión utilizados fueron: artículos a texto completo, sin restricción de idioma, cabe destacar que se incorporo bibliografía de autores clásicos en la materia.

\section{Resultados}

\section{Sobrecarga laboral}

Existe mucha información respecto del impacto que la sobrecarga causa en la atención de salud. Una investigación realizada por Alcalá, Bravo, Expósito y Patrón (2007), demostró que una carga de trabajo que supera la recomendada y requerida por los pacientes en diálisis, es decir 5 pacientes por enfermeras, se traduce en un encubrimiento real de las actividades por parte de equipo de enfermería para satisfacer los requisitos de autocuidado de los pacientes ${ }^{21}$.

Asimismo, otro estudio mostró que elevadas cargas de trabajo, escasa dotación de personal y restricciones de tiempo son factores que afectan el cuidado de los pacientes y el servicio otorgado, así como la capacidad de mantener las habilidades de éstos, lo que sumado a la escasa comunicación, causaba altos niveles de estrés

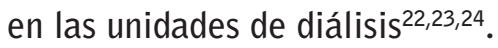


Esta situación es ratificada por Flynn, Thomas-Hawkins \& Clarke en el 2009, quiénes demostraron que altas cargas de trabajo, falta de apoyo en el ambiente de trabajo, se traducen en un deterioro de la calidad de atención y de la satisfacción del usuario, y esto se asociaba con un aumento significativo del SB en las enfermeras; una de cada tres enfermeras registradas presentaba Burnout, un $23.3 \%$ refirió que la carga de trabajo es la causa principal por la cual se cambiarían de trabajo. De las que planificaban dejar su puesto pero permanecer con el empleador, un 46,7\% manifestó el deseo de cambiar de rol a uno que no implicará la atención directa a pacientes, y de éstas un 35,7\% quería cambiarse a un trabajo fuera del centro 0 unidad de diálisis ${ }^{25,44}$.

Además, un estudio de Arikan et al (2007) encontró que las enfermeras de diálisis tienen menor estrés laboral y burnout, y mayor satisfacción laboral, al compararlas con las enfermeras de Unidades de Cuidados Intensivos (UCI) y las de servicios medico-quirúrgicos. Esto se relaciona con otros factores como la edad, años de experiencia y no realizar turnos de noche, pese a que sus horas de trabajo eran las mismas que las enfermeras de UCI.

Entre las características de las enfermeras de las UHD que participaron en el estudio se encontraba que: habían escogido su profesión y las unidades en las que trabajaban; no se habían cambiado de trabajo en los últimos cinco años y presentaban una baja intención de dejar la profesión. Por otra parte, aquellas que llevaban menos de 10 años trabajando tenían mayor estrés laboral, agotamiento emocional y despersonalización, así como la percepción de tener baja realización personal y satisfacción laboral 26,42,43,46.

Otro aspecto importante a mencionar, es la relación entre sobrecarga de trabajo y autonomía; el estudio de Guerrero y Pulido (2010), reveló que las cargas de trabajo eran moderadas en la mayoría de las participantes (76\%): En contraste, la falta de autonomía fue poco reportada, lo que predice un nivel medio de estrés según el modelo de Karasek, el cual postula que el estrés laboral surge cuando las demandas del trabajo son altas, y al mismo tiempo, la capacidad de control de la misma, por falta de recursos es baja ${ }^{48}$. En concordancia con algunos estudios, se encontró sobrecarga y exigencias de atención y concentración. Los principales afectados fueron los hombres (enfer- meros profesionales o auxiliares) y las enfermeras profesionales, que realizan su desempeño profesional en áreas de hospitalización, servicios quirúrgicos, de diálisis y urgencias ${ }^{27}$.

También se encontró un perfil diferente según el rol que desempeñan. Las auxiliares de enfermería presentaban un mayor riesgo extralaboral debido a que tenían problemas económicos, conflictos familiares y debían realizar trabajos en su hogar; no así las enfermeras profesionales quienes tenían peores condiciones de carga por trabajo remunerado, ya sea en la jornada laboral del principal lugar de trabajo o por jornadas part-time. $Y$ a diferencia del personal auxiliar, el mayor riesgo de carga extralaboral fue por requerir de capacitación continua ${ }^{27}$.

Lo anterior, es la antesala para la presencia de tensiones entre familia y trabajo, ya que la carga que adicionan los roles extralaborales junto con las exigencias laborales, dan como resultado procesos de spillover. Este consiste en emociones surgidas cuando un rol permean al otro, procesos de rol (exigencias incompatibles, sobrecarga de rol por exigencias de recursos, experiencias positivas) y procesos de socialización (habilidades, valores y actitudes que se aprenden en un rol y que se manifiestan en el otro) ${ }^{27}$.

\section{Frecuencia y cantidad de tiempo dedicado a la aten- ción de usuarios}

El tiempo de contacto con los pacientes y la complejidad de éstos, genera en el personal de enfermería altos niveles de Burnout ${ }^{28,29}$. En efecto, en un estudio de corte transversal, después de ajustar los resultados por características de las enfermeras y del hospital encontraron que cada paciente adicional a lo establecido estuvo asociado a un $23 \%$ de incremento en la probabilidad de que presenten SB, y un $15 \%$ de incremento en la insatisfacción laboral ${ }^{30}$.

Otra variable que influyó en la presencia de SB fueron las horas de trabajo semanal. Se encontró una relación proporcional, a mayor cantidad de horas trabajadas, aumenta la presencia de síntomas de SB, lo que fue estadísticamente significativo. Un 50\% de síntomas en los profesionales que trabajaban 44 horas o menos, y un $80 \%$ para los que trabajan más de 60 horas semanales. Al analizar el número y porcentaje de personas que tuvieron alta puntuación (en rango de 
SB) por categoría, se observa un alto porcentaje de personas con resultados positivos para las categorías de agotamiento emocional y fuentes de tensión en el trabajo. El valor cercano al $30 \%$ obtenido para trastornos conductuales no es despreciable, ya que involucra todo lo relacionado con abuso de sustancias y conductas de riesgo ${ }^{12}$.

Situación que ratifican los autores Arikan, Köksal \& Gökce (2007), quienes sostienen que más de 49 horas semanales de trabajo, producen en las enfermeras la percepción de menor éxito personal y satisfacción laboral que aquellas que trabajaban entre 40-48 horas semanales. Asimismo, las enfermeras que trabajaban en sistema de turnos rotatorios (incluidos los de noche), tenían mayor estrés laboral, agotamiento emocional y despersonalización y menor percepción de éxito personal y satisfacción laboral, que aquellas que trabajaban en jornada diurna 26,39 .

\section{Niveles de estrés en el equipo de diálisis}

Este predictor es complejo, ya que presenta diversas aristas, así como la relación única enfermera/paciente que existe en las unidades de diálisis puede desdibujar los límites que generalmente existen en la relación tradicional, ya que el tiempo de interacción entre ambos es mayor; por lo tanto, mantener los límites en la relación interpersonal implica mayores desafíos y estrés para las enfermeras de las UHD, que para las enfermeras de otros servicios ${ }^{15,22}$.

Esta relación enfermera/paciente si bien es terapéutica, no está exenta de dependencia afectiva ${ }^{15}$ y de violencia laboral y, en particular, el acoso psicológico emerge como otro factor psicosocial de alto impacto en el grupo ocupacional de enfermeras y auxiliares de enfermería, situación que se reporta en el estudio de Guerrero y Pulido (2010), cuyos hallazgos resultan preocupantes debido a las secuelas emocionales, cognitivas, familiares y de rendimiento laboral. Los grupos más afectados fueron de enfermeras profesionales, que devengan mayor salario y están asignadas a estas unidades ${ }^{27}$.

La complejidad de los pacientes tuvo gran influencia en todos los resultados de las enfermeras, se aprecia que a mayor complejidad: menor satisfacción laboral, mayor estrés, rol en tensión y amenaza laboral. Esto conlleva un impacto en los pacientes, debido a que el estrés laboral de las enfermeras se asoció a una disminución en la capacidad funcional de los pacientes médico-quirúrgicos al alta ${ }^{31,42}$. También influye la imagen profesional que proyecta la enfermera en las personas que atiende. Las enfermeras que pensaban que los pacientes tenían una visión negativa de la profesión, tenían mayor estrés laboral, burnout y bajos niveles de satisfacción; lo que refleja la importancia para las enfermeras de su interacción con los pacientes ${ }^{26}$.

La literatura reporta que la presencia de SB en el personal de salud se relaciona con la escasa participación en la toma de decisiones, información escasa o ambigua que se relaciona directamente con la insuficiente posibilidad de influir en los directivos.

En UHD, la relación intra e inter disciplinar en hemodiálisis es vista como tranquila y positiva por la mayoría de las enfermeras y esto es consistente con encuestas y otros instrumentos aplicados en hospitales en USA, que indican que cerca del $80 \%$ de las enfermeras consideran que las relaciones entre ellas y médicos son entre buenas a excelentes ${ }^{32,45}$.

Asimismo, otro estudio que evaluaba la relación profesional médico-enfermera, encontró que las enfermeras que recibían opiniones positivas de colegas, pacientes y enfermeras coordinadoras, tenían menores niveles de estrés, agotamiento emocional y despersonalización, así como una mejor percepción de éxito personal y satisfacción laboral, que aquellas a las que se evaluaban como moderada o pobre ${ }^{26,39,41}$.

Otro aspecto que da cuenta de la posibilidad de influir en la toma de decisiones con respecto a la atención del pacientes y a su rol profesional, es el efecto de la condición laboral sobre el bienestar del personal de enfermería, los trabajadores con contrato laboral o de planta se ven más afectados y presentan mayor desgaste en su salud por problemas derivados de la definición de roles (principalmente ambigüedad y conflicto) y sentir que no son considerados en la toma de decisiones que afectan directamente su cargo $26,27,42,46$.

Es conocido que el uso de modelos de gestión organizacional promueven el empoderamiento, la autonomía, la toma de decisiones y la satisfacción laboral de las enfermeras; lograr que ésta sea capaz de asumir mejor el rol de la creación, actualización y coordinación de los cuidados y la adquisición de cambios, de manera 
de que exista una mayor consistencia en los cuidados del paciente, dado que cuentan con una mejor definición de los roles y de las tareas asignadas a cada profesional $33,41,42$.

Si bien los modelos de gestión son relevantes para mantener niveles de satisfacción laboral elevado y de productividad en las enfermeras ${ }^{41}$, no menos importante es el recurso humano, situación que es reportada en el estudio de Nayeri, Nazari, Salsali, \& Ahmadi (2005). Este estudio encontró que las enfermeras identifican entre los factores que influencian su efectividad laboral, la evaluación sistemática de los miembros del equipo, un proceso de selección basado en criterios verificables, adecuada comunicación entre el equipo de salud y los supervisores, selección correcta de nuevos integrantes que logren integrarse al equipo actual y provisión de nuevos integrantes con nivel de especialidad acorde a las exigencias del servicio. La ausencia de algunos de estos factores, afecta negativamente la productividad de los equipos de enfermería ${ }^{34}$.

Abundando en lo anterior, otro estudio cuyo objetivo fue relacionar el apoyo de la organización a los servicios de enfermaría y cual eran los efectos de este apoyo en el personal, mostró importantes niveles de insatisfacción laboral y SB. El porcentaje de enfermeras con puntuaciones de SB por encima de las normas publicadas (alrededor del 20\%) para el personal sanitario, varió de $54 \%$ en las enfermeras encuestadas en Pennsylvania/ USA a 34\% en Escocia. Además, las enfermeras que reportaban escaso apoyo para el cuidado de enfermería fueron dos veces más propensas a reportar insatisfacción con sus puestos de trabajo y tener puntuaciones de burnout por encima de la norma ${ }^{26}$.

\section{Complejidad tecnológica y continua capacitación}

Las enfermeras de diálisis están expuestas a los desafíos que presentan las nuevas tecnologías; el grado de estrés que estas generan, depende de su magnitud, del momento en que se presentan y del impacto que genera su uso. Estas se convierten en estresores por si mismas, ya que su implementación requiere de capacitación, generan temor acerca de las implicaciones que esto tendrá para el propio trabajo, la disponibilidad o no de las habilidades necesarias para trabajar con esa nueva tecnología, y las posibilidades de aprender y adquirir los conocimientos necesarios para su uso. A esto último se suma la ansiedad por manejar perfectamente esta tecnologías en un breve tiempo, ya que debe ser usada en la terapia del pacientes ${ }^{35,37}$.

En relación a lo anterior, un estudio, realizado en el Hospital General de Zacatecas, México, que incluyó un total de 336 personas de las cuales 120 eran médicos y 216 enfermeras mostró que el uso de nuevas tecnologías y las actividades de capacitación se relacionaban con el agotamiento emocional. Esto se ve acentuado en quienes muestran mayor cansancio, los que perciben como excesivo el uso de nuevas tecnologías y en aquellos que consideran insuficientes la capacitación ${ }^{36}$.

Asimismo, el uso de tecnologías conlleva un trabajo repetitivo, es así que una investigación reportó puntajes sensiblemente mayor para enfermería, y una de las causas podría ser la excesiva demanda que implica el trabajar con tecnología que le impide la implementación de actividades independientes inherentes al rol de enfermería, que son las que promueven la creatividad en el trabajo $0^{37}$.

\section{Riesgos laborales del personal de diálisis asociados a la terapia dialítica}

Un estudio realizado por Mesa, Gálvez, Franch, Gómez, García, Vázquez et al (2006) mediante el método del Instituto Nacional de Seguridad e Higiene en el Trabajo (I.N.S.H.T), el cual consiste en categorizar los riesgos a los cuales se encuentran expuestos los enfermeros de diálisis, según la probabilidad de ocurrencia y sus consecuencias, categorizándolos en 5 niveles desde un riesgo trivial a un riesgo intolerable; destacó los riesgos del sobreesfuerzo y de exposición a agentes químicos y biológicos, siendo los últimos, los que mayor probabilidad tenían de provocar un accidente laboral. EI sobre esfuerzo se debe a la movilización de pacientes y a la manipulación de fluidos corporales que ocasionan fatiga física y mental ${ }^{38}$.

Guerrero y Pulido (2010), analizaron la relación que existe entre riesgo y protección de los trabajadores del área de la salud, donde las enfermeras profesionales con mayor salario, más jóvenes, fueron las más afectadas. Los principales problemas de salud reportados por el grupo de participantes fueron, en orden: defectos visuales $(35 \%)$, problemas gástricos $(32 \%)$, várices (28\%), síntomas osteomusculares (dolor de espalda $57 \%$, de cintura 50\%, de cuello $49 \%$ ), y síntomas de desgaste psicológico y cansancio emocional (dolor de cabeza $63 \%$ y cansancio mental $45 \%)^{27,39,46}$. 


\section{Conclusiones y sugerencias}

La Organización Mundial de la Salud en el año 2001, reconoció el SB como riesgo ocupacional para profesionales del área de la salud, educación y servicios asistenciales, que produce malestar físico, psíquico y que compromete los resultados en el trabajo, repercutiendo en las organizaciones debido a las ausencias, aumento de conflictos interpersonales y aumento de la rotación de personal ${ }^{40,45}$.

Esta revisión confirma que existen predictores de satisfacción laboral, estrés relacionado con el trabajo y SB. Entre los hallazgos relevantes se encontró que en el profesional de enfermería, disminuye el estrés laboral y el retiro anticipado del rol asistencial cuando alcanza altos niveles de satisfacción al interior de su lugar de trabajo. Además, las relaciones interprofesionales colaborativas, el trabajo en equipo, el respeto al conocimiento y a la experiencia clínica por parte de los directivos, junto a la posibilidad de participar y tomar de decisiones informadas son factores percibidos por las enfermeras/os como protectores de insatisfacción laboral y $\mathrm{SB}^{39,41}$.

Al contrario, la sobrecarga laboral con jornadas de trabajo extensas, es decir, superiores a 9 horas, en una misma unidad de diálisis o servicio clínico, con dedicación exclusiva a la atención de pacientes y sin posibilidad de espacios de esparcimiento, son sin duda considerados como factores de aumentan el SB. Y si a lo anterior, se le agrega la carga afectiva a la que el profesional de enfermería está expuesto durante la jornada de trabajo, la exposición a agentes químicos y la constante incorporación de nuevas tecnologías, conocimientos y responsabilidades, se traduce en un ambiente hostil precursor de SB.

A modo de sugerencia, parece relevante contar con medidas preventivas concretas que tiendan a fomentar el apoyo entre los profesionales y técnicos en la realización de las tareas, por ejemplo, potenciando el trabajo en equipo y la comunicación efectiva. Ello puede reducir o eliminar la precepción de bajo apoyo social y escaso refuerzo positivo. EI incremento de las oportunidades para aplicar los conocimientos y destrezas para el aprendizaje y el desarrollo de nuevas habilidades, a través de la capacitación continua, lo que aumenta las posibilidades de desarrollo 26,46 .
Por otro lado, incentivar la autonomía en la realización de las tareas, acercando tanto como sea posible la ejecución al diseño de las tareas y a la planificación de todas las dimensiones del trabajo, por ejemplo, potenciando la participación efectiva en la toma de decisiones relacionadas con los métodos de trabajo, puede reducir la sensación de baja influencia ${ }^{42}$.

Proporcionar toda la información necesaria, adecuada y a tiempo para facilitar la realización de tareas y la adaptación a los cambios, puede reducir o eliminar la exposición a la baja previsibilidad.

Por último, frente a los riesgos del personal, Mesa et al (2006) propusieron la formación e información sobre la movilización de pacientes y cargas para disminuir el riesgo de sobreesfuerzo; la utilización de guantes de protección y precaución durante la manipulación, para disminuir el riesgo de exposición a agentes químicos y la utilización de las medidas de precaución estándar (mascarilla, guantes, batas, gafas) al entrar en contacto con agentes biológicos ${ }^{38}$.

El principal aporte de este trabajo fue mostrar una realidad en salud que viven los trabajadores del área de diálisis y otras altamente tecnificadas, cuyas implicancias son importantes tanto para la persona, como para los empleadores y los pacientes que requieren de personal de salud en excelentes condiciones de salud mental para otorgar cuidados de alta calidad, por lo que debería ser considerada en las estrategias de prevención internas de las organizaciones.

EI SB por tratarse de una enfermedad multifactorial, requiere una intervención multidisciplinaria, que contemple la participación activa del afectado y de su entorno laboral.

\section{Agradecimientos}

Este trabajo ha sido financiado por la Dirección de Investigación Escuela de Enfermería Pontificia Universidad Católica a través del Concurso Apoyo a la Publicación de Artículos (APA 2010).

Recibido: 27 Noviembre 2011

Revisado: 29 Diciembre 2011

Modificado: 20 Enero 2012

Aceptado: 6 Febrero 2012 


\section{Bibliográficas}

1. Muñoz JC. Síndrome de burnout en unidades de cuidados intensivos. Enferm Intensiva. 2007; 18(1): 1-2. 46.

2. Maslach, C. Burned-out. Human Behavior. 1976; 5, 16-22.

3. Ayala, P. Maslach, C. Characteristics of staff burnout in mental health settings. Hospital \& Community Psychiatry, Vol 29(4), Apr 1978, 233-237.

4. Maslach, C. Schaufeli, W. \& Marek, T. Professional Burnout" Recent Development in Theory and Research. 1993 Taylor \& Francis.

5. Gil-Monte, P. y Peiró, J. Desgaste psíquico en el trabajo. Edit. Síntesis Psicología. España. 1997.

6. Maslach, C. y Pines, A. (1977). "The Burnout Syndrome in the day care setting. Child Care Quarterly. $\mathrm{N}^{\circ}$ 06. San Francisco.

7. Cherniss, C. (1990) natural recovery from burnout: Results of 10-years follow-up study. Journal of health and Human Resources Administration. New York.

8. Maslach, C. y Jackson, S. (1981). Maslach Burnout Inventory ( $2^{\mathrm{a}}$ ed.). Palo Alto, CA.: Consulting Psychologist Press. California.

9. Álvarez, E. y Fernández, L. El Síndrome de «Burnout» 0 el desgaste profesional. I: Revisión de estudios. Revista de la Asociación Española de Neuropsiquiatría. 991, vol. 11, 39. España.

10. Benevides-Pereira AMT, organizador. Burnout: quando o trabalho ameaça o bem estar do trabalhador. [org.] São Paulo: Casa do Psicólogo; 2002. Burnout: o processo de adoecer pelo trabalho. Cap. 2.

11. Goméz M MN, Dodino CN, Aponte CF, Caycedo CE, Riveros MP, Martinez M del PM, et al. Relación entre perfil psciológico, calidad de vida y estrés asistencial em personal de enfermería. Univ Psychol Bogotá. 2005;4(1):63-75.
12. Ordenes D Nadia. Prevalencia de Burnout en trabajadores del hospital Roberto del Río. Rev. chil. pediatr. [revista en la Internet]. 2004 0ct [citado 2011 Dic 17] ; 75(5): 449-454. Disponible en: http://www.scielo. $\mathrm{cl} /$ scielo.php?script=sci_arttext\&pid=S037041062004000500006\&lng=es. doi: 10.4067/ S0370-41062004000500006.

13. García-Huidobro Diego, Spröhnle Carolina, Sapag Jaime. Cuidar a los cuidadores: Un desafío para los profesionales de la salud. Rev. méd. Chile [revista en la Internet]. 2008 Jun [citado 2011 Dic 17]; 136(6): 809-81l. Disponible en: http://www.scielo.cl/scielo.php?script=sci_arttext\&pid=S003498872008000600019\&lng=es. doi: 10.4067/ S0034-98872008000600019.

14. Trucco $M$, Valenzuela $P$, Trucco D. Estrés ocupacional en personal de salud. Rev Méd Chile 1999; 127: 1453-61.

15. Barrios S. Enfermeras y Pacientes: Cara a Cara Cronica. Revista Asodi. 2010;21(88):5-7.

16. Albadejo, R. Villanueva, R. Síndrome de Burnout en el personal de enfermería de un hospital de Madrid. Revista Española de Salud Pública.78 (2004), 505-516.

17. Decreto $\mathrm{N}^{\circ} 2357$ y sus modificaciones. Reglamento sobre Centros de Diálisis. Diario Oficial 31.12.94. http://www.seremisalud9.cl/Accion_Sanitaria/ docs/legislacion/ds_2357\%20centros\%20dialisis. pdf.

18. Hernández, V, Palacios, G, Rubio, FJ, Porras, G, Rodríguez, J, Uguet, $M$, et al. Evaluación del grado de dependencia de los pacientes en Hemodiálisis Mediante Test Delta. Comunicaciones XXXI Congreso Nacional de la Sociedad Española de Enfermería Nefrológica. 2006 0ct; 31:371-381.

19. Peña P, López J, de la Torre $L$, Muelas F. Cargas de trabajo en una unidad de nefrológica. Comunicaciones XVII Congreso Nacional de la Sociedad Española de Enfermería Nefrológica. 1992 Nov;17.

20. Poldinger, P. "Depresiones larvadas, depresiones por agotamiento y síndrome de burnout". En "EI 
enfermo psicosomático en la práctica". 1997, Editorial Herder.

21. Alcalá, C., Bravo, M., Expósito, M., Patrón, E. Tiempos Modernos. Presentado en el $21^{\circ}$ congreso nacional de la asociación española de Enfermería de anestesia, reanimación y terapia del dolor; 2007 0ct 24, 25 y 26; León, España.

22. Dermody, K. y Bennett, PN. Nurse stress in hospital and satellite haemodialysis units. Journal of Renal Care, 34: 28-32. 2010 doi: 10.1111/j.1755-6686 .2008 .00007 .

23. Grau G, Chacón M: Burnout: una amenaza a los equipos de salud. Conferencia $2^{a}$ Jornadas de Actualización en Psicología de la salud. Asociación Colombiana de Psicología de la Salud. 1998.

24. Olivar C, González S, Martínez MM: Factores relacionados con la satisfacción laboral y el desgaste profesional en los médicos de atención primaria de Asturias. Aten Primaria 1999; 24: 352-9.

25. Flynn L, Thomas-Hawkins C, Clarke SP. Organizational traits, care processes, and burnout among chronic hemodialysis nurses. Western journal of nursing research. 2009; 31(5):569.

26. Arikan, F., Köksal, C. \& Gökce C.Work-related stress, burnout, and job satisfaction of dialysis nurses in association with perceived relations with professional contacts Dialysis \& Transplantation 2007, Wiley Online Library [Internet]. [citado 2011 Nov 19];Available from: http://onlinelibrary.wiley.com/ doi/10.1002/dat.20119/pdf.

27. Guerrero J y Pulido G. Trabajo, salud y régimen contractual en personal de enfermería: un enfoque psicosocial. Av enferm, 2010 XXVIII(2): 111-122.

28. Atance JC: Aspectos epidemiológicos del síndrome de Burnout en personal sanitario. Rev Esp Salud Pública 1997; 71: 293-303.

29. Norrie P: Do intensive care staff suffer more stress than staff in other care environment? A discussion. Intensive and Critical care Nursing 1995; 50: 1071-6.

30. Aiken, L., Clarke, S., Sloane, D., Sochalski, J. \& Silber, J. Hospital nurse staffing and patient morta- lity, nurse burnout, and job dissatisfaction. JAMA: the journal of the American Medical Association. 2002; 288(16):1987-1993.

31. McGillis, L. \& Doran, D. A Study of the impact of nursing staff mix models and organizational change strategies on patient, system and nurse outcomes. The Newsletter of the Canadian Health Services Research Foundation. 2001; 1:28-32.

32. NurseWeek/AONE. (2002). National survey of registered nurses. Retrieved October 31, 2002, from www.nurseweek.com/survey/.

33. Harwood L., Ridley J. \& Lawrence-Murphy J.A. et al. (2007) Nurses' perceptions of the impact of a renal nursing professional practice model on nursing outcomes, characteristics of practice environments and empowerment - Part II. CANNT Journal (online). Available at: http://www.highbeam.com/doc/ 1P3-1295622591.html, accessed 9 August 2011.

34. Nayeri, N., Nazari, A., Salsali, M., Ahmadi, F. Iranian staff nurses'views of their productivity and human resource factors improving and impeding it: a Qualitative study. Human Resources for Health. 2005; 3(9):1-11.

35. Apiquian A. El síndrome de burnout en las empresas. Presentación realizada en el Tercer Congreso de Escuelas de Psicología de las Universidades Red Anáhuac. Mérida, Yucatán 26 y 27 de abril, 2007, recuperado de http://www.idconline.com.mx/labo$\mathrm{ral} /$ pdfs-laboral/El\%20burnout $\% 20$ en $\% 20 l a s \% 20$ empresas.pdf.

36. Rojero, MG., Ruiz de Chávez, D., Pando, M., Aranda, C., Almeida, C. Presencia del síndrome de burnout en médicos y enfermeras del hospital general ISSSTE zacatecas y factores que lo asocian, recuperado de http://factorespsicosociales.com/tercerforo/trabajos/documentos/TL07.2.pdf.

37. Camponovo Meier, 0., \& Morín Imbert, P. (2000). Síndrome de burnout en el personal de salud de un hospital público de la ciudad de Rosario. Investigación en Salud, 3(1-2), 73-93.

38. Mesa E, Gálvez A, Franch B, Gómez C, García JA, Vázquez MD, et al. Condiciones, riesgos laborales 
y medidas correctoras del puesto de trabajo en los enfermeros de diálisis. Comunicaciones XXVI Congreso Nacional de la Sociedad Española de Enfermería Nefrológica. 2006 Sept; 26:160-166.

39. Klersy C., Callegari A., Martinelli V., Vizzardi V., Navino C., Malberti F. et al. Burnout in health care providers of dialysis service in Northern Italy-a multicentre study Nephrol Dial Transplant (2007) 22: 2283-2290. doi:10.1093/ndt/gfmlll.

40. World Health Organisation (WHO) World Health Report 2001 [texto en internet]. Geneve; 2001. [citado 2011 dic. 17]. Disponível em: http://www. who.int/report.

41. Aiken, L., \& Patrician, P.A. (2000). Measuring organizational traits of hospitals: The revised nursing work index. Nursing Research, 49(3), 146-153.

42. Laschinger, Heather K. Spence; Judith Shamian,; Donna Thomson,. "Impact of Magnet Hospital Characteristics on Nurses' Perceptions of Trust Burnout Quality of Care, And Work Satisfaction.(Statistical Data Included)." Nursing Economics. Jannetti Publications, Inc. 2001. HighBeam Research. 20 Dec. $2011<$ http://www.highbeam.com>.

43. Sadovich JM. Work excitement in nursing: an examination of the relationship between work excitement and burnout. (55).Nurs Econ. 2005;23(2):91-6. [PubMed].

44. Cohen-Katz J, Wiley SD, Capuano T, Baker DM, Kimmel S, Shapiro S. The effects of mindfulnessbased stress reduction on nurse stress and burnout, Part II: A quantitative and qualitative study. Holist Nurs Pract. 2005;19 (1):26-35.

45. Thomas-Hawkins C, Denno M, Currier H, Wick G. Staff nurses' perceptions of the work environment in freestanding hemodialysis facilities. Nephrology Nursing Journal. 2003; 30(2):169-183.

46. Ridley, J., Wilson, B., Harwood, L., \& Laschinger, H. (2009). Work environment, health outcomes and magnet hospital traits in the Canadian nephrology nursing scene. Canadian Association of Nephrology Nurses and Technologies Research Grant Journal, 19(1), 28-35.

47. Önder C., Basim N. Examination of developmental models of occupational burnout using burnout profiles of nurses, Journal of Advanced Nursing, 2008, 64, 5.

48. Karasek, R. Demad/control model: a social, emotional and phisiological approach to stress risk and active behavior development. En: Encyclopedia of Occupational Health and Safety (pp 34.6). Sweden; Organización Internacional del Trabajo, 2008. 